\title{
Patient Experience With Fatigue and Qualitative Interview-Based Evidence of Content Validation of The FACIT-Fatigue in Systemic Lupus Erythematosus
}

\author{
Kimberly Raymond (1) - Josephine Park · Ashish V. Joshi • \\ Michelle K. White
}

Received: October 29, 2020 / Accepted: February 16, 2021 / Published online: March 9, 2021

(C) The Author(s) 2021

\section{ABSTRACT}

Introduction: Fatigue is highly prevalent and burdensome in systemic lupus erythematosus (SLE). The Functional Assessment of Chronic Illness Therapy-Fatigue (FACIT-Fatigue) is a patient-reported questionnaire that measures physical and mental fatigue and consequent impact on daily living. Qualitative evidence of content validity in SLE is limited. This study (GSK Study 209226) assessed the content validity of the FACIT-Fatigue for SLE and explored patients' experiences of SLE-related fatigue using qualitative methods.

Methods: Fatigue-related themes were identified through semi-structured, hybrid cognitive debriefing and concept elicitation interviews and evaluated for concordance with the FACIT-Fatigue. Results: Fatigue was experienced regularly by all participants ( $N=15,86.7 \%$ female) and was rated as the most bothersome symptom of SLE by $11 / 15$ participants. All participants reported emotional impacts of fatigue, while 14/15 and 9/15 participants also reported impacts on social life and physical functioning, respectively. Most (12/15) reported that fatigue

K. Raymond $(\varangle) \cdot$ M. K. White

QualityMetric, Johnston, RI, USA

e-mail: kraymond@qualitymetric.com

J. Park · A. V. Joshi

GlaxoSmithKline, Value Evidence and Outcomes,

Collegeville, PA, USA interfered with their ability to fulfill work- or school-related roles, and activities of daily living were limited in all participants. All (14/14) reported that a meaningful change in their level of fatigue would be the ability to have a more active and normal lifestyle. Concept mapping showed that all 13 FACIT-Fatigue items mapped directly onto concepts spontaneously mentioned by participants. Cognitive debriefing revealed that $13 / 15$ participants found the instructions easy to understand and $11 / 15$ participants endorsed the recall period (7 days) as appropriate. Participants found the FACIT-Fatigue items were clear and relevant. Most participants (11/ 15) reported that all response options adequately captured their experience of fatigue.

Conclusions: Qualitative evaluation of the content validity of the FACIT-Fatigue supports it as an appropriate measure for assessing the impact of fatigue on daily living of patients with SLE. The tool is easily understood by patients and a valuable resource for measuring a common and debilitating symptom of this condition.

Keywords: Content validity; FACIT-Fatigue; Fatigue; Patient experience; SLE; Quality of life 


\section{Key Summary Points}

Physical and mental fatigue can be assessed by the Functional Assessment of Chronic Illness Therapy-Fatigue (FACIT-Fatigue) scale. Currently, there is limited evidence on how valid the content of this questionnaire is to assess fatigue experienced by patients with systemic lupus erythematosus (SLE).

This study investigated patients' experience with fatigue related to SLE, and whether the FACIT-Fatigue is an appropriate questionnaire to evaluate fatigue in patients with this disease.

This study highlighted several fatigue-related themes that are important to patients with SLE, and its findings support the use of the FACIT Fatigue as an easily understood method of assessing the burden from fatigue in patients with SLE.

\section{DIGITAL FEATURES}

This article is published with digital features, including a summary slide, to facilitate understanding of the article. To view digital features for this article go to https://doi.org/10.6084/ m9.figshare.13713916.

\section{INTRODUCTION}

Systemic lupus erythematosus (SLE) is a complex autoimmune disease with a range of clinical manifestations [1] and periods of relative remission of disease activity followed by exacerbations or flares (increases in symptoms or severity of symptoms) [2]. The estimated incidence of SLE in the United States is 5.1/100,000 per year, and the prevalence is $52.2 / 100,000$; females and those of non-white race are more likely to be affected [3]. SLE can be life-threatening when major organs are impacted. More frequently, it presents with a number of chronic and debilitating non-specific symptoms [1].

Fatigue is one of the most common symptoms and is experienced by the majority of patients with SLE $[4,5]$. It has been linked to worse quality of life, depression, increased work disability, and increased health care costs [6-8]. As fatigue imposes a high burden in patients with SLE, it is important that it can be accurately assessed to ensure that patients can be effectively monitored and managed. Moreover, both the United States Food and Drug Administration (FDA) and the European Medicines Agency recommend measuring fatigue during clinical trials of treatments for SLE $[9,10]$.

Despite the importance of measuring fatigue in SLE, there is no consensus on what instruments should be used, and no instrument has been developed specifically for patients with SLE [9]. The Functional Assessment of Chronic Illness Therapy-Fatigue (FACIT-Fatigue) is a 13-item patient-reported outcome (PRO) instrument designed to capture multiple aspects of fatigue [11]. Since its development in 1997 for use in patients with cancer, its appropriateness for use in other diseases has been evaluated, including autoimmune conditions like rheumatoid arthritis; and it has been used in numerous SLE clinical trials [12]. Systematic reviews of PRO measures indicated that the FACIT-Fatigue has good face and psychometric validity, and contains concepts appropriate for measuring fatigue in patients with SLE $[13,14]$. Content validity of the FACIT-Fatigue has been previously established through evidence reported in studies of patients with SLE [13-15], as well as evidence collected through focus groups with patients with SLE [16]. The current study expands on existing evidence of content validity of the FACIT-Fatigue by collecting data directly from patients through individual oneon-one qualitative interviews. This approach allowed more in-depth information to be gathered on patients' experience of fatigue to assess the appropriateness of the FACIT-Fatigue for use in measuring fatigue in an SLE population. 


\section{Objectives}

The purpose of the present qualitative study was to assess the content validity of the FACITFatigue using a hybrid approach of concept elicitation (CE) and cognitive debriefing (CD) in individual interviews. The study also aimed to gain a better understanding of patients' experiences of living with the condition and managing the symptoms of SLE.

\section{METHODS}

\section{Study Design}

This qualitative study (GSK study 209226) was designed to evaluate the content validity of the FACIT-Fatigue for use within the SLE population. The design is in accordance with the FDA Guidance for Industry: Patient-Reported Outcome Measures: Use in Medical Product Development to Support Labeling Claims and the International Society for Pharmacoeconomics and Outcomes Research (ISPOR) Clinical Outcome Assessment Emerging Good Practices Task Force Guidance on Assessing Respondent Understanding of PRO Instruments [17, 18]. The study approach used hybrid CE and CD interviews of patients diagnosed with SLE. The CE component aimed to gain insight into the symptoms experienced by patients living with SLE, and how these symptoms impact daily life including experience and impacts of fatigue. This information was used to better understand whether the concepts covered by the FACITFatigue are relevant and appropriate to measure fatigue in those with SLE. The CD component aimed to determine whether the FACIT-Fatigue is relevant and easily understood by participants. Interviews were conducted by telephone in July 2018.

\section{Study Participants}

The sample size of 15 participants was determined based on industry best practice regarding the number of interviews needed to reach saturation of a concept, i.e., the point at which additional interviews are not expected to yield additional information [18-20]. Participants were screened and recruited from across the USA via an existing panel provided by a global research company specializing in healthcare data collection. The following inclusion criteria were applied: adults aged $\geq 18$ years with a selfreported doctor's diagnosis of SLE for $\geq 6$ months and who self-reported to testing positive for antinuclear antibodies or anti-doublestranded deoxyribonucleic acid, who reported persistent SLE symptoms in the past 6 months or self-reported at least one SLE flare in the past 12 months despite treatment with steroids and/ or an immunosuppressant, and who had the ability to complete the interview in fluent English. Participants were selected, based on self-reported screening questions, to ensure a diverse range of disease severity, number of flares, and types of symptoms commonly experienced in SLE. Ethics approval was granted by the New England Independent Review Board (120180137) and participants provided written informed consent prior to the interview, with consent confirmed verbally at the start of each interview. The study was performed in accordance with the Declaration of Helsinki 1964 and its later amendments.

\section{Interview Procedure}

Individual interviews (90 min, by phone) were conducted by an experienced qualitative scientist with a semi-structured interview guide. Feedback from early interviews was incorporated into subsequent interviews to verify early emerging themes.

During the CE interview component, participants were asked open-ended questions to investigate their daily experience of SLE-related fatigue and its impact on their daily life. Following the CE component, participants completed the FACIT-Fatigue using a think-aloud $\mathrm{CD}$ approach, in which participants were asked to verbalize their thoughts as they worked through the FACIT-Fatigue, articulating how they made sense of instructions, items, and response choices $[18,21]$. Participants were also asked to discuss what would be a meaningful 
change in their symptoms of fatigue as a result of treatment for SLE. The CD interview component was designed to assess the FACITFatigue for relevance, appropriateness, comprehensiveness, and the ability to be easily understood and completed by participants with SLE. Participants were also asked to note any items or aspects of the FACIT-Fatigue that they found confusing, and these were discussed with the interviewer retrospectively upon completion. Following the think-aloud, participants were asked structured questions on the relevance, comprehensiveness, and clarity of the FACITFatigue and were prompted to comment on the format, instructions, items, recall period, and response choices.

\section{Data Analysis}

Interviews were audio-recorded and transcribed, and final transcripts were anonymized to ensure any personal health information was excluded. Data were then coded using a grounded theory approach [22]. The grounded theory approach allows for themes to emerge from the data rather than imposing a priori hypotheses [22]. Content coding was completed by two qualitative analysts and a qualitative research scientist. The coding tasks for the CE and CD sections of the interviews were each completed by one qualitative analyst and reviewed by another, to support consistency. The qualitative research scientist reviewed the final coding structure and all codes. Any identified discrepancies between coders were reviewed, discussed, and resolved by the research team to help ensure the reliability of the coding.

All coding and data analysis of the CE component were carried out using NVivo, version 11.0 [23]. The first batch of five interviews was coded by both analysts to establish a preliminary coding structure; these five interviews were then recoded using the agreed set of codes. An analysis of saturation was conducted with the data from the CE component of the interview to provide evidence that enough interviews were completed to fully understand concepts important to participants. The coding process for saturation analysis involved developing a preliminary set of first- level codes by reviewing transcripts $1-5$. This review facilitated an understanding of the data and helped determine which themes were emerging as most common across participants. After discussion of the first batch of transcripts, the study coding team (qualitative analysts and qualitative research scientist) reached consensus on the final set of codes with which to proceed. Concepts produced spontaneously were given preference in theme identification. Next, interviews 1-5 were recoded for second-level codes and then coded again using the entire list of new codes. Interviews $6-10$ were then coded using the final list of codes. Finally, interviews 11-15 were coded to confirm saturation, ensuring that no additional, relevant concepts emerged. Following completion of the coding and data analysis, item mapping was conducted to demonstrate whether there is correspondence between the fatigue-related concepts identified in the CE interviews and the FACIT-Fatigue scale.

Narrative data from the CD portions of interviews were coded in Excel using a series of ratings to summarize feedback. Each transcript was reviewed and coded for content related to instructions, recall period, the relevance and comprehension of each item and the item response options of the FACIT-Fatigue. Items were assigned two codes: one for whether a problem was reported and a second for whether it was a spontaneous or prompted remark. A pre-specified threshold of $\geq 4$ participants having a consistent comment on a particular item was set to guide conclusions, recommendations, and determinations regarding item relevance and comprehension and determining whether a reported problem warranted a change. Every reported problem, however, was evaluated by the research team to determine whether the requested change was an individual preference or style-related request or a true problem with comprehension or responding to an item.

\section{RESULTS}

\section{Participant Characteristics}

A total of 15 participants with SLE were interviewed. The mean (standard deviation $[\mathrm{SD}]$ ) age 
Table 1 Patient demographics and characteristics

Total population $(N=15)$

Age, years, mean (SD)

$52.1(13.1)$

Female, $n$ (\%)

$13(86.7)$

Race, $n$ (\%)

Non-Hispanic/white

$8(53.3)$

Black/African American

$6(40.0)$

Other

Years since SLE diagnosis, mean (SD)

$17.9(11.2)$

Patient-reported SLE severity, $\boldsymbol{n}$ (\%)

Moderate

Severe

Don't know/not sure

My doctor has not told me

Number of flares in the previous 12 months, $n$ (\%)
$1-3$
$6(40.0)$
$4-6$
$4(26.7)$
$7-10$
$2(13.3)$
$\geq 11$
$3(20.0)$

$S D$ standard deviation, $S L E$ systemic lupus erythematosus. All information in this table was derived through patient selfreporting

was $52.1(13.1)$ years and 13 participants $(86.7 \%)$ were female (Table 1). Almost half $(7 / 15,46.7 \%)$ of the participants interviewed self-reported moderate SLE, and 4/15 (26.7\%) self-reported severe SLE. All participants self-reported at least one flare in the previous 12 months, with $9 / 15$ $(60.0 \%)$ reporting $\geq 4$ flares in this period (Table 1).

\section{Concept Elicitation}

Saturation analysis showed that $90 \%$ of the high-frequency themes emerged by the tenth interview and retained their prevalence over the course of analysis, suggesting that additional interviews would not have resulted in further themes or sub-themes. Three late-emerging themes related to emotional impacts were reviewed by the research team to determine whether new information was introduced that warranted additional data collection. It was determined that these individuals had described emotional impacts that were similar to those of other participants using different words, were minor, an outlier, or technically unrelated, and did not warrant further data collection.

Overall, 27 signs and symptoms associated with SLE were reported, affecting a range of bodily systems and functions. Fatigue, pain, sleep disturbance, and cognitive issues were reported by all participants (Fig. 1). Almost all participants $(14 / 15,93.3 \%)$ reported that their symptoms could be brought on or intensified by triggers. Participants most commonly noted exposure to the sun/photosensitivity $(12 / 15$, $80.0 \%)$ and physical exertion $(10 / 15,66.7 \%)$ as triggers (Table 2). The majority of participants 


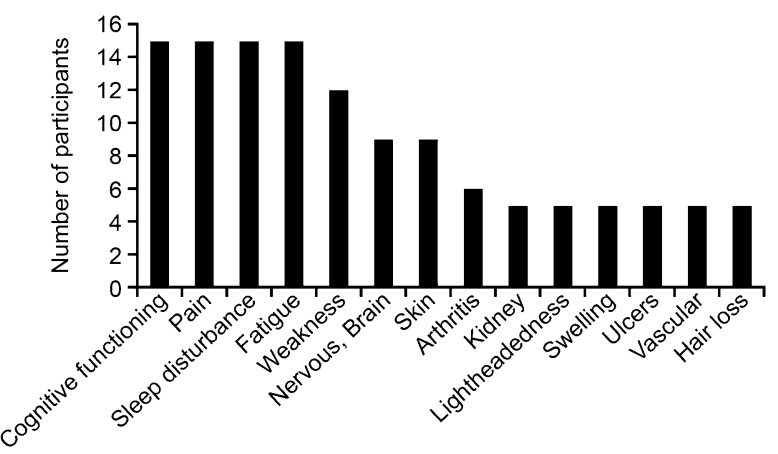

Fig. 1 Commonly reported SLE symptoms*. *Only symptoms reported by at least five participants are shown. $S L E$ systemic lupus erythematosus

Table 2 Symptom triggers and most bothersome symptoms

Total population

$(N=15)$

Triggers reported to prompt or intensify symptoms, $n(\%)$

Exposure to sun/
photosensitivity

$12(80.0)$

Physical exertion

$10(66.7)$

Stress

$5(33.3)$

Cold weather

$3(20.0)$

Stopping medication

2 (13.3)

Diet

2 (13.3)

Symptoms reported as the most bothersome, $n$ (\%)

$\begin{array}{ll}\text { Fatigue } & 11(73.3) \\ \text { Pain } & 3(20.0) \\ \text { Difficulty concentrating } & 1(6.7) \\ \text { Hair loss } & 1(6.7) \\ \text { Insomnia } & 1(6.7)\end{array}$

Participants may have reported multiple symptom triggers and multiple symptoms as bothersome; therefore, the percentages shown in this table could exceed 100\%; all information in this table was derived through patient selfreporting

$(9 / 15,60.0 \%)$ reported that the severity of their symptoms and frequency and intensity of flares varied over time.
Fatigue was reported as the most bothersome symptom by $11 / 15 \quad(73.3 \%)$ participants (Table 2). All reported regularly experiencing fatigue, with most feeling fatigued every day $(12 / 15,80.0 \%)$ and others a few days a week $(3 / 15,20.0 \%)$. The majority $(12 / 15,80.0 \%)$ also reported that their fatigue often fluctuates over the course of the day, or from day to day. Of 13 participants who described experiencing flares, ten $(76.9 \%)$ reported intense fatigue associated with their flares.

Participants were asked to describe how fatigue impacted their quality of life (Table 3). Physical functioning was reported to be impacted by $9 / 15(60.0 \%)$ participants. They emphasized that physical difficulties affected their employment and physical inactivity impacted their ability to maintain a healthy weight. All participants reported emotional impacts of fatigue, where the most common was frustration/stress due to being unable to lead a "normal" life and accomplish tasks. Almost all participants $(14 / 15,93.3 \%)$ reported ways in which fatigue had profoundly impacted their social lives. The most frequently reported social functioning difficulty was being unable to participate in social activities. Most participants $(12 / 15,80.0 \%)$ reported that fatigue interfered with their ability to fulfil work- or school-related roles. Additionally, activities of daily living were limited in all participants, who reported difficulty starting/finishing tasks. The majority $(14 / 15,93.3 \%)$ were unable to do much other than rest or sleep due to fatigue.

All participants (14/14 [100\%]) who answered the question on what a meaningful change in their fatigue would be reported that the ability to be more active and have a more normal lifestyle would have the most meaningful and important impact on their lives.

Concept mapping showed that all 13 items of the FACIT-Fatigue mapped directly onto concepts spontaneously mentioned by participants during the interviews without major gaps (Table 4). 
Table 3 Representative quotes from participants asked to describe impacts of fatigue

\begin{tabular}{|c|c|c|}
\hline Impact & $\begin{array}{l}\text { Number } \\
(\%) \\
\text { of } \\
\text { participants }\end{array}$ & Representative quote $[$ sex, age in years] \\
\hline Physical functioning & $9(60.0)$ & \multirow{6}{*}{$\begin{array}{l}\text { You know, doctors tell me, "Oh, you need to lose weight." I'm a plus } \\
\text { size woman. And I have to lose weight, but if I try to walk or run } \\
\text { or anything, I'm wiped out in a matter of seconds. So, I stay } \\
\text { sedentary most of the time. If I had more energy, I would love to } \\
\text { go to an exercise class. I know I need to lose weight, but my body is } \\
\text { telling me "no" and I don't know what to do for it to say "yes." } \\
\text { [Female, Age 42] }\end{array}$} \\
\hline Difficulties walking & $5(33.3)$ & \\
\hline Difficulties exercising & $3(20.0)$ & \\
\hline Difficulties lifting and carrying things & $3(20.0)$ & \\
\hline Climbing & $2(13.3)$ & \\
\hline Standing & $2(13.3)$ & \\
\hline Emotional impacts & $15(100.0)$ & \multirow{7}{*}{$\begin{array}{l}\text { I get very frustrated when someone wants to do something, and you } \\
\text { have to say, "Eh, I'm not up to it," or "That's too much walking," } \\
\text { or you know, whatever the case may be. [Female, Age 44] }\end{array}$} \\
\hline Frustration/stress & $14(93.3)$ & \\
\hline Sadness/depression & $11(73.3)$ & \\
\hline Loss of motivation & $10(66.7)$ & \\
\hline Hopelessness/resignation & $8(53.3)$ & \\
\hline Loneliness/alienation & $6(40.0)$ & \\
\hline Fear/anxiety & $5(33.0)$ & \\
\hline $\begin{array}{l}\text { Social relationships/functioning } \\
\text { impacts }\end{array}$ & $14(93.3)$ & \multirow{4}{*}{$\begin{array}{l}\text { The fatigue impacts my life because in a way that I'm not as open or } \\
\text { I'm not as eager to go out and do things when people or family } \\
\text { and friends invite me to do something. So, especially if I haven't } \\
\text { seen or talked to my friends in a while. I have friends that I've been } \\
\text { friends with since high school. We all try to get together once a } \\
\text { month just to keep in touch. I'm hesitant to do any type of social } \\
\text { activity just because of the fatigue. [Female, Age 40] }\end{array}$} \\
\hline $\begin{array}{l}\text { Unable to participate in social } \\
\text { activities }\end{array}$ & $14(93.3)$ & \\
\hline $\begin{array}{l}\text { Overestimation/misunderstanding of } \\
\text { participants' energy by others }\end{array}$ & $9(60.0)$ & \\
\hline Relationship issues & $6(40.0)$ & \\
\hline Role functioning impacts & $12(80.0)$ & \multirow{3}{*}{$\begin{array}{l}\text { I don't do anything anymore because I'm either too tired or I hurt } \\
\text { too much. The last job I worked, I was working in an assisted- } \\
\text { living home and they wanted me to come in and I was just in too } \\
\text { much pain and too tired to come in so I told them no. It has a very } \\
\text { profound effect on me and I'm sure I'm not the only one. Just the } \\
\text { tiredness alone, it's very frustrating. [Female, Age 60] }\end{array}$} \\
\hline $\begin{array}{l}\text { Trouble focusing/staying awake at } \\
\text { work }\end{array}$ & $7(46.7)$ & \\
\hline $\begin{array}{l}\text { Taking time off to stay at home and } \\
\text { rest }\end{array}$ & $6(40.0)$ & \\
\hline Impacts on activities of daily living & $15(100.0)$ & \multirow{4}{*}{$\begin{array}{l}\text { You do laundry and everything. I don't finish it. I'm like, "keep the } \\
\text { clothes in the dryer," or something. I might take them out and } \\
\text { don't fold them up. So that's what I mean [when I say that I have] } \\
\text { trouble finishing things. [Female, Age 43] }\end{array}$} \\
\hline Difficulty starting/finishing tasks & $15(100.0)$ & \\
\hline $\begin{array}{l}\text { Unable to do much other than rest/ } \\
\text { sleep }\end{array}$ & $14(93.3)$ & \\
\hline Disrupted eating habits & $7(46.7)$ & \\
\hline
\end{tabular}




\section{Cognitive Debriefing}

Overall, the FACIT-Fatigue was easy to complete and understand by participants and was comprehensive for capturing the symptoms and impacts of fatigue related to SLE. The thinkaloud CD method revealed that 13 participants found the instructions easy to understand and 11 endorsed the recall period (7 days) as appropriate. FACIT-Fatigue items were generally found to be clear and relevant to patients' experiences with fatigue. No items received four or more common concerns from study participants (Table 5) and follow-up queries ensured that any concern reported by participants did not interfere with their ability to understand or respond to an item.

Due to the complexity of SLE and its unique presentation, two participants wanted the response options to capture more detail, such as adding comment boxes to enable elaboration with open-ended responses. However, most participants $(11 / 15,73.3 \%)$ reported that the response options were adequate to capture their experience of fatigue for each item.

\section{DISCUSSION}

Participants in this study described fatigue as the most debilitating symptom of their condition. These findings confirm fatigue as an important concept to patients with SLE and suggest the value of including it as a secondary endpoint in randomized controlled trials and longitudinal observational studies to measure efficacy of SLE treatments [14]. Participants also confirmed the importance, relevance, and comprehensiveness of concepts covered in the FACIT-Fatigue. They reported that the instrument accurately captures the type of fatigue they experience as part of their condition, including the range of physical, emotional, and social impacts of SLE-related fatigue in daily life. These results build on previous studies and provide additional qualitative evidence of content validity of the FACIT-Fatigue for use in patients with SLE.

Although previous studies have investigated the content validity of the FACIT-Fatigue in SLE
$[13,15,16]$, the current study was the first investigation to assess content validity using individual in-depth qualitative interviews. Findings from the current study parallel evidence gathered in a previous study that used focus groups, lending further support to the FACIT-Fatigue as relevant for measuring fatigue in patients with SLE [16]. This study also expanded on the prior work by Kosinski et al., who reported that patients found two items of the FACIT-Fatigue ("I'm too tired to eat" and "I have energy") less relevant than other items. The time spent with each individual in our study allowed for deeper probing into the meaning of these items and the relevance of the concepts to the participants.

While Kosinski et al. found that only a small number of patients were physically too tired to eat due to the effort required for meal preparation [16], nearly all participants in the current study $(14 / 15,93.3 \%)$ reported that the item "I'm too tired to eat" covered a relevant and important aspect of SLE-related fatigue. Indepth probing on these items revealed that most participants $(12 / 15,80.0 \%)$, interpreted the item as intended, namely as the act of eating rather than food preparation. A subset of these participants $(5 / 12,41.7 \%)$ also indicated that preparing food would be a separate item for which they might provide a different response, further demonstrating their interpretation of the original item as the physical act of eating. Many participants also reported difficulty maintaining a healthy diet because SLE-related fatigue interfered with both eating and meal preparation.

The item "I have energy" was also reported in Kosinski et al. to be less relevant to patients with SLE, as most patients were continually tired and without energy [16]. Individual interviews in the current study allowed for additional inquiry of those participants who reported " 0 - not at all" to this item during the cognitive debriefing exercise. Most participants $(14 / 15,93.3 \%)$ described this item as relevant and easy to understand, and all reported experiencing times when they did have energy. Those who had responded " 0 - not at all" on this item also reported experiencing moments when their energy levels fluctuated and were 
Table 4 Concept mapping of the FACIT-Fatigue

\section{Item content}

I feel fatigued

I feel weak all over

I feel listless (“washed out”)

I feel tired

I have trouble starting things because I am tired

I have trouble finishing things because I am tired

I have energy

I am able to do my usual activities

I need to sleep during the day
Representative quote [sex, age in years]

All the time. I'm always fatigued. You know what? Sometimes you feel like if this is going to be my life, um, what kind of life is this? [Female, Age 53]

Um, I guess weak. Weak would be-weak is kind of a-because your muscles feel heavy, and you're too weak to lift them up. You know. It's not weakness-it wouldn't be a weakness without the fatigue, you know. Um, for instance, I was watching TV one time when I was fatigued, and I wanted to change the channel, and I remember telling my arm to reach out and get the control. You know. I had to-normally, I would just-my brain would say: change channel, you just reach out and get the control. And I had to say: I need to move my arm out there and get that control. [Female, Age 58]

Um, I feel that sometimes and I think with me that's what the whole mentally washed out comes in. Listless, doesn't have anything to do with your body per se, but your mind. [Female, Age 39]

Whereas, I mean, I can be going and doing something, then all of the sudden I just get so tired that I have to stop what I'm doing, sit down, and just rest. [Female, Age 64]

There was this little project about with my daughter's and her step-son, uh, he's autistic and I'm trying to help gather information for them you know for them to see about getting, treating him right, meeting his healthy needs. And I was working on it this morning and I just had to, I just had to, no actually before last night and I just had to drop it. See, there's the memory issue. Um, I just had to drop it and go on to bed because I was just so tired and in so much pain. [Female, Age 60]

You do laundry and everything. I don't finish it. I'm like, "keep the clothes in the dryer," or something. I might take them out and don't fold them up. So that's what I mean like trouble finishing things." [Female, Age 43]

Every once in a while, I'll have a day where I'm full of energy. - that's very few and far between. [Female, Age 35]

When you have lupus fatigue, opening up that Little Debbie package is sometimes difficult. You know. And a lot of times when I'm fatigued, I'm glad we talked about this, because I'd forgotten about this. I will get up and go out into the kitchen and say: I need to eat. And I'll walk to the refrigerator and open the refrigerator and stand there and look in the refrigerator. And then close the refrigerator and then walk to the pantry. And then look in the pantry. And just kind of go: nope. Can't handle any of that. [Female, Age 58]

I need to sleep during the day, quite a bit. Um, usually, you know, at least once a day, sometimes more-more than that. Sometimes I just run out of gas. [Female, Age 53] 
Table 4 continued

\begin{tabular}{|c|c|}
\hline Item content & Representative quote [sex, age in years] \\
\hline I am too tired to eat & $\begin{array}{l}\text { I have had times where I've had to decide, “OK, do I want to sleep or do I want } \\
\text { to eat?" And I've been too tired and I will go to sleep. So, it has happened. } \\
\text { [Female, Age 42] }\end{array}$ \\
\hline I need help doing my usual activities & $\begin{array}{l}\text { Um, I haven't been able to keep up with the yard. I had to have my son come } \\
\text { and mow the yard and it had gotten so high before he got it mowed that it } \\
\text { has to be mowed again. And, um, what else has he done for me this week? } \\
\text { He's done several things for me this week that usually I would've taken care } \\
\text { of myself. [Female, Age 58] }\end{array}$ \\
\hline $\begin{array}{l}\text { I am frustrated by being too tired to do } \\
\text { the things I want to do }\end{array}$ & $\begin{array}{l}\text { You know, I'm always like, I'm just, I'm just frustrated with the disease, period. } \\
\text { [Female, Age 53] }\end{array}$ \\
\hline $\begin{array}{l}\text { I have to limit my social activity because I } \\
\text { am tired }\end{array}$ & $\begin{array}{l}\text { Yeah, and that's-I mean, I just don't-my-my social activity is very limited. If } \\
\text { I-I do have friends, and I know every once in a while-every once in a while, } \\
\text { I want to go kind of hang out and do something, it's going to be cut to next } \\
\text { to nothing. I have a friend that asked me to come hang out. He was going to } \\
\text { go have a drink, and I wanted to know-if I'd go with him. And as opposed } \\
\text { to, you know, wanting to go out to a restaurant and have a drink and sit } \\
\text { there and talk, we ended up going to, you know, the corner bar that's a block } \\
\text { away, because I-it was close to home, and in case something went wrong, I } \\
\text { could be close to home. [Male, Age 46] }\end{array}$ \\
\hline
\end{tabular}

FACIT Functional Assessment of Chronic Illness Therapy

closer to what they considered normal. Nine of these participants described energy levels that fluctuated over a week or a day, and two described their energy as self-motivated. Thus, despite findings of previous studies to the contrary, this study supports the relevance of these two items (eating and energy) as important and relevant to patients experiencing SLE-related fatigue.

Overall, cognitive interviews in the current study demonstrated that participants had no problem understanding the instructions, items, or response choices of the FACIT-Fatigue, and found the recall period to be acceptable. Although some difficulties were reported in interpreting items, no consistent problems or suggestions were reported by participants that interfered with their ability to understand or respond to an item. Of the minimal problems reported, many were from an 85-year-old participant whose SLE-related symptoms may have also been confounded by other age-related symptoms.

Like most qualitative research in rare diseases, conclusions drawn from the current study are based on a small sample size. Additionally, given the higher prevalence of SLE in females $[24,25]$ there were difficulties in recruiting male participants. However, while formal analyses were not conducted, the experience of fatigue and the responses from the two male participants were not noticeably different from those of the female participants. Another limitation is that the diagnosis of SLE, the number of flares patients experienced in the past 12 months, and severity were all self-reported by participants during screening rather than derived through disease activity indices or clinician confirmation. Potential misclassification relating to reported diagnosis was minimized by requesting information known only to those with SLE. Furthermore, almost all participants (14/15) 
Table 5 Patient comments per item of the FACIT-Fatigue from cognitive debriefing

\begin{tabular}{|c|c|c|}
\hline Item & $\begin{array}{l}\text { Number of participants reporting } \\
\text { problems (participant number, sex, age } \\
\text { in years) }\end{array}$ & Nature of problem \\
\hline I feel fatigued & 1 (Participant 12 - Male, Age 85) & Difficulty interpreting the term "fatigued" \\
\hline I feel weak all over & $\begin{array}{l}2 \text { (Participant } 07 \text { - Female, Age 60; } \\
\quad \text { Participant } 12 \text { - Male, Age 85) }\end{array}$ & $\begin{array}{l}\text { Difficulty attributing weakness to lupus vs. other } \\
\text { conditions }\end{array}$ \\
\hline $\begin{array}{l}\text { I feel listless (“washed } \\
\text { out") }\end{array}$ & 1 (Participant 07 - Female, Age 60) & $\begin{array}{l}\text { Difficulty understanding terms: "listless" and } \\
\text { "washed out" }\end{array}$ \\
\hline I feel tired & $\begin{array}{l}2 \text { (Participant } 02 \text { - Female, Age 42; } \\
\quad \text { Participant } 12 \text { - Male, Age 85) }\end{array}$ & Always tired \\
\hline $\begin{array}{l}\text { I have trouble starting } \\
\text { things because I am tired }\end{array}$ & 0 & $\mathrm{~N} / \mathrm{A}^{*}$ \\
\hline $\begin{array}{l}\text { I have trouble finishing } \\
\text { things because I am tired }\end{array}$ & 0 & $\mathrm{~N} / \mathrm{A}^{*}$ \\
\hline I have energy & 1 (Participant 12 - Male, Age 85) & Difficulty interpreting the term "energy" \\
\hline $\begin{array}{l}\text { I am able to do my usual } \\
\text { activities }\end{array}$ & $\begin{array}{l}3 \text { (Participant } 08 \text { - Female, Age 35; } \\
\text { Participant } 12 \text { - Male, Age 85; } \\
\text { Participant } 13 \text { - Female, Age 44) }\end{array}$ & $\begin{array}{l}\text { While individuals were able to comprehend } \\
\text { "usual activity," three participants conveyed } \\
\text { that a "usual activity" might be different for } \\
\text { them, were it not for their SLE diagnosis }\end{array}$ \\
\hline $\begin{array}{l}\text { I need to sleep during the } \\
\text { day }\end{array}$ & 1 (Participant 12 - Male, Age 85) & $\begin{array}{l}\text { Difficulty discerning whether he needs more sleep } \\
\text { during the day or in bed }\end{array}$ \\
\hline I am too tired to eat & 1 (Participant 12 - Male, Age 85) & $\begin{array}{l}\text { Difficulty interpreting whether he was being } \\
\text { asked about the actual act of eating }\end{array}$ \\
\hline $\begin{array}{l}\text { I need help doing my usual } \\
\text { activities }\end{array}$ & $\begin{array}{l}3 \text { (Participant } 08 \text { - Female, Age 35; } \\
\text { Participant } 12 \text { - Male, Age 85; } \\
\text { Participant } 13 \text { - Female, Age 44) }\end{array}$ & $\begin{array}{l}\text { While individuals were able to comprehend } \\
\text { "usual activity," three participants conveyed } \\
\text { that a "usual activity" might be different for } \\
\text { them, were it not for their SLE diagnosis }\end{array}$ \\
\hline $\begin{array}{l}\text { I am frustrated by being } \\
\text { too tired to do the } \\
\text { things I want to do }\end{array}$ & 1 (Participant 12 - Male, Age 85) & Frustrated by more than just being tired \\
\hline $\begin{array}{l}\text { I have to limit my social } \\
\text { activity because I am } \\
\text { tired }\end{array}$ & 1 (Participant 12 - Male, Age 85) & $\begin{array}{l}\text { Difficulty differentiating social limitations due to } \\
\text { fatigue versus other symptoms of lupus }\end{array}$ \\
\hline
\end{tabular}

FACIT Functional Assessment of Chronic Illness Therapy, N/A not applicable, SLE systemic lupus erythematosus

${ }^{*}$ Indicates that there were no reported problems related to clarity or relevance for this item 
indicated during screening that they had received a positive antinuclear antibody test. Both the range of severity levels reported during screening and the wide array of symptoms and impacts revealed during interviews confirmed the inclusion of multiple levels of severity in this sample. While our aim was to ensure the FACIT-Fatigue was appropriate to capture fatigue across different levels of severity, as experienced by the patient, we also recognized that the absence of a validated tool in this study suggests that reported levels may not parallel physician assessment of patient disease activity. Discrepancies between patient and physician assessment of SLE disease activity are well documented [26]; and thus, participants in this study could have overestimated the level of severity. Furthermore, four out of 15 participants reported that they did not know their level of severity. Other clinical characteristics such as medication use and existing comorbidities were not collected. Therefore, any potential impact of medication use and conditions comorbid to SLE on reported levels of fatigue is unknown and should be acknowledged as a limitation. In order to test the English version of the FACIT-Fatigue, the sample was limited to those who read and spoke fluent English. Further research would therefore be necessary to confirm that the items would be as well understood in a less-fluent English-speaking or non-English-speaking population. Additionally, information on socio-economic status was not collected for this sample. It is possible that facets of socio-economic status, including income and education level, could impact understanding of the FACIT-Fatigue items.

Despite these limitations, this study used a sound and rigorous methodological approach to explore the primary research questions. Qualitative interviews were conducted on an individual level, which allowed for detailed data to be collected without responses being influenced by other participants. It also allowed for in-depth probing to expand on and clarify findings from previous studies. Additionally, the exploratory nature of the CE component facilitated the uncovering of the most important concepts related to participants' experience of fatigue in SLE; and the think-aloud approach of the CD component confirmed relevance of the concepts included in the FACIT-Fatigue and participants' understanding of the instrument items.

\section{CONCLUSIONS}

The results of this study provide qualitative evidence to support the FACIT-Fatigue as an appropriate measure for assessing fatigue and its impacts on health-related quality of life in patients with SLE. The instrument measures fatigue-related concepts relevant to SLE, using items that are easily understood. Fatigue has been shown to be the most commonly reported symptom of SLE, greatly impacting how patients are able to function in their day-to-day life, warranting more standardized use of measures that are able to capture both the essence of this fatigue and its impacts. Furthermore, the results support the use of the FACIT-Fatigue in SLE clinical trials and other research exploring fatigue in this population.

\section{ACKNOWLEDGEMENTS}

We thank the participants of the study. Analytical support was provided by April Mitchell, and administrative support was provided by Sean O'Connor and Nishtha Baranwal, of QualityMetric.

Funding. Funding This study (209226) was funded by GSK. Employees of QualityMetric were not paid for manuscript development. The Rapid Service Fee was funded by GSK.

Authorship. All named authors meet the International Committee of Medical Journal Editors (ICMJE) criteria for authorship for this article, take responsibility for the integrity of the work as a whole, and have given their approval for this version to be published.

Authorship Contributions. KR, JP, and MKW were responsible for the concept or design of the study, acquisition of data, and data analysis or interpretation. AVJ was 
responsible for the concept or design of the study and data analysis or interpretation. KR, JP, AVJ, and MKW read and approved the final manuscript for submission.

Medical writing and Editorial Assistance. Medical writing support was provided by Casmira Brazaitis, PhD, and Jennie McLean, $\mathrm{PhD}$, of Fishawack Indicia Ltd, UK, part of Fishawack Health, and was funded by GSK.

Disclosures. Josephine Park and Ashish V. Joshi are employees of GSK and hold stock in the company. Kimberly Raymond and Michelle K. White are employees of QualityMetric and have nothing to disclose.

Compliance with Ethics Guidelines. Ethics approval was granted by the New England Independent Review Board (120180137) and participants provided written informed consent prior to the interview, with consent confirmed verbally at the start of each interview. The study was performed in accordance with the Declaration of Helsinki 1964 and its later amendments.

Data Availability. The datasets generated and/or analyzed during the current study are not publicly available due to the unavailability of the GSK database link for a study that does not test a GSK drug, but they are available from the corresponding author on reasonable request.

Open Access. This article is licensed under a Creative Commons Attribution-NonCommercial 4.0 International License, which permits any non-commercial use, sharing, adaptation, distribution and reproduction in any medium or format, as long as you give appropriate credit to the original author(s) and the source, provide a link to the Creative Commons licence, and indicate if changes were made. The images or other third party material in this article are included in the article's Creative Commons licence, unless indicated otherwise in a credit line to the material. If material is not included in the article's Creative Commons licence and your intended use is not permitted by statutory regulation or exceeds the permitted use, you will need to obtain permission directly from the copyright holder. To view a copy of this licence, visit http://creativecommons.org/licenses/by$\mathrm{nc} / 4.0 /$.

\section{REFERENCES}

1. D'Cruz DP. Systemic lupus erythematosus. BMJ. 2006;332(7546):890-4.

2. Petri M, Genovese M, Engle E, Hochberg M. Definition, incidence, and clinical description of flare in systemic lupus erythematosus. A prospective cohort study. Arthritis Rheum. 1991;34(8):937-44.

3. Danchenko N, Satia JA, Anthony MS. Epidemiology of systemic lupus erythematosus: a comparison of worldwide disease burden. Lupus. 2006;15(5): 308-18.

4. Cojocaru M, Cojocaru IM, Silosi I, Vrabie CD. Manifestations of systemic lupus erythematosus. Maedica (Buchar). 2011;6(4):330-6.

5. Sterling K, Gallop K, Swinburn P, Flood E, French A, Al Sawah S, et al. Patient-reported fatigue and its impact on patients with systemic lupus erythematosus. Lupus. 2014;23(2):124-32.

6. Figueiredo-Braga $\mathrm{M}$, Cornaby C, Cortez A, Bernardes M, Terroso G, Figueiredo M, et al. Depression and anxiety in systemic lupus erythematosus: the crosstalk between immunological, clinical, and psychosocial factors. Medicine (Baltimore). 2018;97(28):e11376.

7. Bexelius C, Wachtmeister K, Skare P, Jonsson L, Vollenhoven R. Drivers of cost and health-related quality of life in patients with systemic lupus erythematosus (SLE): a Swedish nationwide study based on patient reports. Lupus. 2013;22(8): 793-801.

8. Basta F, Margiotta DPE, Vadacca M, Vernuccio A, Mazzuca C, Picchianti Diamanti A, et al. Is fatigue a cause of work disability in systemic lupus erythematosus? Results from a systematic literature review. Eur Rev Med Pharmacol Sci. 2018;22(14): 4589-97.

9. Food and Drug Administration. Guidance for industry: systemic lupus erythematosus - developing medical products for treatment. 2010; Available from: www.fda.gov/downloads/Drugs/Guidance ComplianceRegulatoryInformation/Guidances/ucm 072063.pdf. (Accessed Jul 2019) 
10. European Medicines Agency. Guidelines on clinical investigation of medicinal products for the treatment of systemic lupus erythematosus and lupus nephritis. 2015. Available from: https://www.ema. europa.eu/en/documents/scientific-guideline/guide line-clinical-investigation-medicinal-products-treat ment-systemic-lupus-erythematosus-lupus_en.pdf. (Accessed Jul 2019)

11. FACIT.org. The functional assessment of chronic illness therapy-fatigue (FACIT-F) Scale: summary of development and validation. Available from: www. facit.org/FACITOrg/Questionnaires. (Accessed Jul 2019)

12. Hewlett S, Dures E, Almeida C. Measures of fatigue: Bristol Rheumatoid Arthritis Fatigue Multi-Dimensional Questionnaire (BRAF MDQ), Bristol Rheumatoid Arthritis Fatigue Numerical Rating Scales (BRAF NRS) for severity, effect, and coping, Chalder Fatigue Questionnaire (CFQ), Checklist Individual Strength (CIS20R and CIS8R), Fatigue Severity Scale (FSS), Functional Assessment Chronic Illness Therapy (Fatigue) (FACIT-F), Multi-Dimensional Assessment of Fatigue (MAF), Multi-Dimensional Fatigue Inventory (MFI), Pediatric Quality Of Life (PedsQL) Multi-Dimensional Fatigue Scale, Profile of Fatigue (ProF), Short Form 36 Vitality Subscale (SF-36 VT), and Visual Analog Scales (VAS). Arthritis Care Res (Hoboken). 2011;63(Suppl 11): S263-86.

13. Holloway L, Humphrey L, Heron L, Pilling C, Kitchen H, Hojbjerre L, et al. Patient-reported outcome measures for systemic lupus erythematosus clinical trials: a review of content validity, face validity and psychometric performance. Health Qual Life Outcomes. 2014;12:116.

14. Strand V, Simon LS, Meara AS, Touma Z. Measurement properties of selected patient-reported outcome measures for use in randomised controlled trials in patients with systemic lupus erythematosus: a systematic review. Lupus Sci Med. 2020;7(1): e000373.

15. Lai JS, Beaumont JL, Ogale S, Brunetta P, Cella D. Validation of the functional assessment of chronic illness therapy-fatigue scale in patients with moderately to severely active systemic lupus erythematosus, participating in a clinical trial. J Rheumatol. 2011;38(4):672-9.

16. Kosinski M, Gajria K, Fernandes AW, Cella D. Qualitative validation of the FACIT-fatigue scale in systemic lupus erythematosus. Lupus. 2013;22(5): 422-30.

17. Food and Drug Administration. Guidance for industry: patient-reported outcome measures: use in medical product development to support labeling claims. 2009. Available from: https://www.fda. gov/downloads/drugs/guidances/ucm193282.pdf. (Accessed 2019 July)

18. Patrick DL, Burke LB, Gwaltney CJ, Leidy NK, Martin ML, Molsen E, et al. Content validity-establishing and reporting the evidence in newly developed patient-reported outcomes (PRO) instruments for medical product evaluation: ISPOR PRO Good Research Practices Task Force report: part 2-assessing respondent understanding. Value Health. 2011;14(8):978-88.

19. Brod M, Tesler LE, Christensen TL. Qualitative research and content validity: developing best practices based on science and experience. Qual Life Res. 2009;18(9):1263-78.

20. Hennink MM, Kaiser BN, Marconi VC. Code saturation versus meaning saturation: how many interviews are enough? Qual Health Res. 2017;27(4):591-608.

21. Ericsson KA, Simon HA. Verbal reports as data. Psychol Rev. 1980;87(3):215-51.

22. Strauss AL, Corbin JM. Basics of qualitative research: Grounded theory procedures and techniques. Newbury Park: Sage Publications; 1990.

23. Wallace DJ, Stohl W, Furie RA, Lisse JR, McKay JD, Merrill JT, et al. A phase II, randomized, doubleblind, placebo-controlled, dose-ranging study of belimumab in patients with active systemic lupus erythematosus. Arthritis Rheum. 2009;61(9): 1168-78.

24. D'Cruz DP, Khamashta MA, Hughes GR. Systemic lupus erythematosus. Lancet. 2007;369(9561): 587-96.

25. Rahman A, Isenberg DA. Systemic lupus erythematosus. N Engl J Med. 2008;358(9):929-39.

26. Golder V, Ooi JJY, Antony AS, Ko T, Morton S, Kandane-Rathnayake $\mathrm{R}$, et al. Discordance of patient and physician health status concerns in systemic lupus erythematosus. Lupus. 2018;27(3): 501-6. 